\title{
Ecología Comunitaria de Metazoos Parásitos del Bonito Sarda chiliensis Cuvier, 1832 (Perciformes: Scombridae) de la Costa Peruana
}

\author{
Community Ecology of Metazoan Parasites of Pacific Bonito Sarda chiliensis \\ Cuvier, 1832 (Perciformes: Scombridae) from the Peruvian Coast \\ Jhon Chero ${ }^{1}$, Gloria Sáez ${ }^{1}$, José Iannacone ${ }^{2,3,5}$, Celso Cruces ${ }^{1}$, Lorena Alvariño ${ }^{2}$, \\ José Luque ${ }^{4}$
}

\section{RESUMEN}

\begin{abstract}
Se analizó la comunidad de metazoos parásitos de $S$. chiliensis de la costa marina peruana. Se recolectaron 100 especímenes de $S$. chiliensis del Terminal Pesquero de Chorrillos, Lima, Perú, entre enero de 2013 y diciembre de 2014 y se realizó la necropsia para estudiar su comunidad de metazoos parásitos. Los cestodos dominaron en riqueza de especies y en porcentaje de individuos colectados $(\mathrm{N}=5 ; 44.6 \%)$, seguido de los nematodos $(\mathrm{N}=4 ; 27.0 \%)$, acantocéfalos $(\mathrm{N}=3 ; 2.3 \%)$, trematodos $(\mathrm{N}=1 ; 23.2 \%)$ y finalmente los copépodos parásitos $(\mathrm{N}=1 ; 3 \%)$. Los endoparásitos dominaron $(97 \%)$ frente a los ectoparásitos (3\%). El 21.4\% del total de parásitos colectados fueron formas maduras de metazoos de localización branquial y gastrointestinal y el 78.6\% correspondió a formas larvarias. Los tres parásitos con mayor importancia específica (prevalencia y abundancia media) de 14 taxas encontradas fueron Sphyriocephalus tergestinus, Anisakis sp2 y Unitubulotestis pelamydis. La longitud total de $S$. chiliensis no presentó asociación entre la prevalencia y abundancia de dos parásitos, a excepción de U. pelamydis que observó relación entre la prevalencia y la talla del pez. La comparación entre los valores de $\mathrm{k}_{\mathrm{n}}$ (factor de condición relativo) entre peces parasitados y no parasitados de $S$.
\end{abstract}

${ }^{1}$ Laboratorio de Parasitología, ${ }^{2}$ Laboratorio de Ecología y Biodiversidad Animal (LEBA), Facultad de Ciencias Naturales y Matemática (FCNNM), Universidad Nacional Federico Villarreal (UNFV), Lima, Perú

${ }^{3}$ Laboratorio de Invertebrados, Facultad de Ciencias Biológicas, Universidad Ricardo Palma (URP), Lima, Perú

${ }^{4}$ Departamento de Parasitologia Animal, Curso de Pós-graduação em Ciências Veterinárias, Universidade Federal Rural do Rio de Janeiro - UFRRJ, Seropédica, Rio de Janeiro, Brasil

${ }^{5}$ E-mail: joseiannacone@gmail.com

Recibido: 6 de febrero de 2016

Aceptado para publicación: 10 de mayo de 2016 
chiliensis, mostró diferencias para los tres parásitos, presentándose mayores valores en los no parasitados. Los valores de diversidad alfa mostraron mayores valores en las hembras que en los machos de $S$. chiliensis. Los valores de Chao-2 indican que en el componente comunitario se requiere incrementar el tamaño de la muestra debido a que se espera encontrar hasta 18 especies (22\% adicionales).

Palabras clave: Anisakis, ecología parasitaria, parásitos de peces, Perú, Sphyriocephalus, Unitubulotestis

\section{Abstract}

Metazoan parasite community of $S$. chiliensis of Peruvian shoreline was analyzed. Specimens of $S$. chiliensis $(\mathrm{n}=100)$ were collected from Fishing Terminal of Chorrillos, Lima, Peru between January 2013 and December 2014. Necropsies were conducted to study their metazoan parasite community. Tapeworms dominated species richness and percentage of collected individuals $(\mathrm{N}=5 ; 44.6 \%)$, followed by nematodes $(\mathrm{N}=4 ; 27.0 \%)$, acanthocephalan $(\mathrm{N}=3 ; 2 \%)$, flukes $(\mathrm{N}=1 ; 23.2 \%)$ and finally the parasitic copepods $(\mathrm{N}=1$, $3 \%$ ). Endoparasites dominated (97\%) against ectoparasites (3\%). The $21.4 \%$ of collected parasites were mature forms of metazoan gill and gastrointestinal location and $78.6 \%$ were larval forms. The three parasites with more specific importance (average prevalence and abundance) of 14 taxa were Sphyriocephalus tergestinus, Anisakis sp2 and Unitubulotestis pelamydis. The total length of S. chiliensis did not presented association between prevalence and abundance of two parasites, except for $U$. pelamydis that showed a relationship between the prevalence and size of the fish. The comparison between the values of $\mathrm{k}_{\mathrm{n}}$ (relative condition factor) between parasitized and non-parasitized $S$. chiliensis showed differences for the three parasites, having higher values in non-parasitized. The values of alpha diversity showed higher values in females than in males of $S$. chiliensis. Values of Chao-2 show that in the community component is required to increase the sample size because was expected to find up to 18 species $(22 \%)$.

Key words: Anisakis, parasite ecology, fish parasites, Peru, Sphyriocephalus, Unitubulotestis

\section{INTRODUCCIÓN}

El bonito, Sarda chiliensis (Cuvier, 1831) (Scombridae), es una especie epipelágica nerítica de la Corriente Costera Peruana que vive formando cardúmenes (Chirichigno y Cornejo, 2001). Son peces de comportamiento principalmente carnívoro que se alimentan de peces pequeños como la anchoveta Engraulis ringens Jenyns, 1842, y de moluscos y crustáceos pelágicos (Collette y Nauen, 1983). Esta especie endémica del Pacífico Oriental es capturada incidentalmente en la pesca comercial y es uno de los recur- sos más importantes para el consumo humano (Collette et al., 2011). Chirichigno y Cornejo (2001) señalan que este tipo de escómbrido se distribuye desde Puerto Pizarro (Perú) a Talcahuano (Chile). Además, es mencionada para Islas Hawái e Islas Galápagos.

La mayoría de estudios realizados en el Perú, con la finalidad de evaluar la fauna parasitaria de $S$. chiliensis y otros escómbridos marinos, han sido de índole mayormente cualitativa o de amplitud de ámbito (Ilave, 1964; Rego et al., 1968; Baer, 1969; Escalante 1983; Lazarte y Córdova, 1987; Ruelas y Córdova, 1988; Luque y Iannacone, 1991; 
Tantaleán y Huiza, 1994; Sarmiento et al., 1999; Tantaleán et al., 2005; Chero et al., 2015). Oliva et al. (2008) y Cruces et al. (2014) realizaron una primera aproximación cuantitativa de la parasitofauna del escómbrido sudamericano Scomber japonicus Houttuyn, 1782 del Puerto del Callao (Lima) y Puerto de Chicama (La Libertad), respectivamente. Posteriormente no se ha efectuado ningún trabajo relacionado a conocer los aspectos ecológicos de la parasitofauna en peces escómbridos en el Perú.

En este trabajo se analiza la comunidad de metazoos parásitos de $S$. chiliensis de la costa marina peruana.

\section{Materiales y Métodos}

Entre enero de 2013 y diciembre de 2014, 100 especímenes de $S$. chiliensis del Terminal Pesquero de Chorrillos, Lima, Perú (12 $09^{\prime} 23$ " S, $77^{\circ} 01$ '55" W) fueron recolectados para estudiar su comunidad de metazoos parásitos. Los peces fueron identificados de acuerdo a Chirichigno y Cornejo (2001). Los 100 especímenes de $S$. chiliensis presentaron una longitud total (LT) entre 32 a $48 \mathrm{~cm}(39.94 \pm 2.98)$, siendo 40.25 $\pm 2.73 \mathrm{~cm}$ en machos $(\mathrm{n}=55)$ y $39.56 \pm 3.25$ $\mathrm{cm}$ en hembras $(\mathrm{n}=45)$. Se empleó la prueba de $t$ de Student para determinar diferencias entre la LT de los peces machos y hembras, cumpliéndose previamente con el requisito de normalidad empleando la prueba de Kolmogorov-Smirnov y homogeneidad de varianza con la prueba de Levene. No se encontró diferencias significativas en la LT entre $\operatorname{sexos}(\mathrm{t}=1.20 ; \mathrm{p}=0.23 ; \mathrm{n}=100)$.

Se realizó la necropsia de los peces, evaluando principalmente piel, aletas, fosas nasales, branquias, opérculo, cavidad bucal, estómago, intestino, riñón, corazón, superficie visceral y cavidad celómica, empleando el microscopio estereoscopio a 10x, y se recolectaron los parásitos. La identificación genérica de los digeneos se realizó usando las claves de Yamaguti (1971), Gibson et al. (2002) y Jones et al. (2005). La identificación genérica de los céstodos se realizó usando la clave de Schmidt (1986) y Heinz y Dailey (1974), en nematodos la clave de Moravec (2006) y para acantocéfalos la clave de Petrochenko (1971) y la lista actualizada de Amin (2013). El material parasitológico colectado se depositó en la Colección Científica de Protozoos y Metazoos Parásitos de la Universidad Nacional Federico Villarreal (CPMP-UNFV).

Los índices ecológicos parasitológicos (prevalencia, abundancia e intensidad media) se calcularon de acuerdo a Bush et al. (1997). Se determinó la prevalencia $(\mathrm{P})$, intensidad media (IM) y abundancia media (AM) para cada uno de los parásitos catastrados en los 100 especímenes de $S$. chiliensis. También se calculó la P y la abundancia total en base al número total de peces parasitados y del total de parásitos para todas las especies encontradas, independiente de su identidad taxonómica, respectivamente. Se usó el índice de importancia específica (I) con el fin de obtener un índice integrado de infección ecológica (Bursey et al., 2001). La frecuencia de dominancia y la frecuencia de dominancia relativa de cada especie parásita se determinó según Rohde et al. (1995).

Para el caso de las especies parásitas con prevalencias mayores al $10 \%$ se realizaron los estadígrafos inferenciales y se calculó el índice de dispersión (ID), que clasifica la distribución en: contagiosa $(>1)$, uniforme $(<1)$ y aleatoria (0) (Iannacone et al., 2008, Iannacone y Alvariño 2009, 2011, 2012).

El coeficiente de correlación de Pearson se usó para determinar la relación entre el tamaño del hospedero (LT) con la abundancia de cada parásito. El coeficiente de correlación de Spearman se empleó para determinar la relación entre la LT del hospedero y la $\mathrm{P}$ de cada parásito. Se aplicó la prueba $\mathrm{X}^{2}$ razón de verosimilitud para tablas de contingencia $2 \times 2$ para determinar el grado de 
Cuadro 1. Prevalencia, intensidad media, abundancia media y sitio de infección de metazoos parásitos de Sarda chiliensis de la zona costera de Chorrillos, Lima, Perú

\begin{tabular}{|c|c|c|c|c|c|}
\hline Parásitos & Estadio & $\begin{array}{c}\text { Prevalencia } \\
(\%)\end{array}$ & $\begin{array}{c}\text { Abundancia } \\
\text { media }\end{array}$ & $\begin{array}{c}\text { Intensidad } \\
\text { media }\end{array}$ & $\begin{array}{l}\text { Sitio de } \\
\text { infección }\end{array}$ \\
\hline \multicolumn{6}{|l|}{ Copépoda } \\
\hline Caligus bonito & adulto & 5 & 0.08 & 1.6 & opérculo \\
\hline \multicolumn{6}{|l|}{ Trematoda } \\
\hline $\begin{array}{l}\text { Unitubulotestis } \\
\text { pelamidys }\end{array}$ & adulto & 17 & 0.62 & 3.65 & estómago \\
\hline \multicolumn{6}{|l|}{ Cestoda } \\
\hline $\begin{array}{c}\text { Sphyriocephalus } \\
\text { tergestinus }\end{array}$ & plerocercoide & 32 & 1.09 & 3.41 & estómago \\
\hline $\begin{array}{l}\text { Tentacularia } \\
\text { coryphaenae }\end{array}$ & plerocercoide & 4 & 0.05 & 1.25 & cavidad corporal \\
\hline Nybelinia spl & plerocercoide & 1 & 0.02 & 2 & cavidad corporal \\
\hline Nybelinia sp2 & plerocercoide & 1 & 0.01 & 1 & cavidad corporal \\
\hline $\begin{array}{c}\text { Adenocephalus } \\
\text { pacificus }\end{array}$ & plerocercoide & 2 & 0.02 & 1 & mesenterio \\
\hline \multicolumn{6}{|l|}{ Nematoda } \\
\hline Anisakis sp 1 & larva & 2 & 0.02 & 1 & mesenterio \\
\hline Anisakis sp 2 & larva & 31 & 0.47 & 1.52 & mesenterio \\
\hline Contracaecum sp & larva & 1 & 0.03 & 3 & mesenterio \\
\hline Hysterothylacium $\mathrm{sp}$ & larva & 2 & 0.20 & 10 & mesenterio \\
\hline \multicolumn{6}{|l|}{ Acantoc ephala } \\
\hline Corynosoma $\mathrm{sp}$ & cistacanto & 3 & 0.04 & 1.33 & cavidad corporal \\
\hline Bolbosoma sp & juvenil & 1 & 0.01 & 1 & cavidad corporal \\
\hline Rhadinorhynchus sp & adulto & 1 & 0.01 & 1 & intestino \\
\hline Total & & 58 & 2.67 & 4.60 & \\
\hline
\end{tabular}

dependencia entre el sexo del hospedero y la P. El efecto del sexo en la AM de infección parasitaria se evaluó con la prueba de $\mathrm{t}$ de Student.

El factor de condición relativo $\left(\mathrm{K}_{\mathrm{n}}\right)$ fue calculado en base al W y LT del hospedero, a partir de la fórmula: $\mathrm{K}_{\mathrm{n}}=\mathrm{w} / \mathrm{W}^{*}$, donde: $\mathrm{W}=$ peso del pez $(\mathrm{g}) \mathrm{y} \mathrm{W}^{*}=$ peso calculado por regresión de la ecuación LT-W previamente calculada (Longart et al., 2011). Se empleó la prueba de t de Student para comparar los valores de $\mathrm{K}_{\mathrm{n}}$ entre los peces parasitados y no parasitados para cada especie de helminto. 


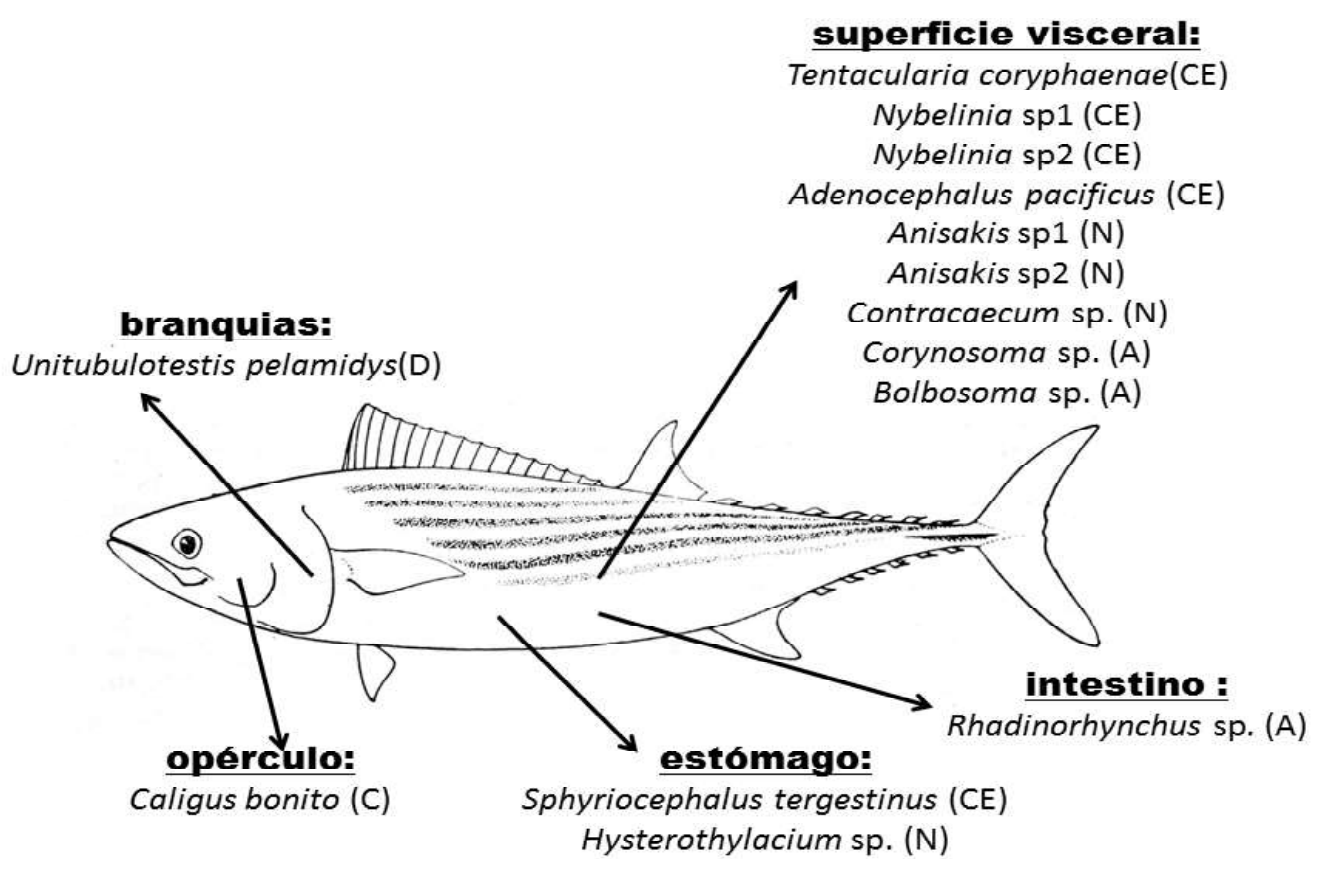

Figura 1. Espécimen de Sarda chiliensis (Scombridae). Helmintos agrupados en sitios de infección. ( $\mathrm{N}=$ Nematoda; $\mathrm{D}=$ Digenea; $\mathrm{CE}=$ Cestoda; $\mathrm{A}=$ Acantocephala; $\mathrm{C}=$ Copepoda)

Se determinaron los índices de diversidad alfa: Riqueza, Individuos, Simpson, Shannon, Brillouin, Menhinick, Margalef, Equitabilidad, Berger-Parker y Chao-2 (Moreno, 2001; Bego y Von Zuben, 2010) para el componente comunitario parasitario, para machos y para hembras. Se calculó el dendrograma con el índice cuantitativo de Morisita para comparar la similitud de los parásitos compartidos entre los peces catastrados. El análisis de los índices de diversidad y el estimador de riqueza de parásitos se realizaron con la ayuda del programa PAST (Paleontological Statistics software) v. 2012, 2.16. El nivel de significancia fue evaluado a un alfa $=0.05$. Para el cálculo de las pruebas estadísticas descriptivas e inferenciales se usó el paquete estadístico IBM SPSS Statistics 21.0 - 2010.

\section{Resultados}

Se colectó un total de 267 parásitos, con una abundancia media total de 2.67. El promedio de la riqueza de especies fue 1.05 (14). Los cestodos dominaron en riqueza de especies y en porcentaje de individuos colectados $(\mathrm{N}=5 ; \mathrm{n}=119 ; 44.6 \%)$, seguido de los nematodos $(\mathrm{N}=4 ; \quad \mathrm{n}=72 ; \quad 27.0 \%)$, acantocéfalos $(\mathrm{N}=3 ; \mathrm{n}=6 ; 2.3 \%)$, trematodos $(\mathrm{N}=1 ; \mathrm{n}=62 ; 23.2 \%)$ y finalmente $10 \mathrm{~s}$ copépodos parásitos $(\mathrm{N}=1 ; n=8 ; 3.0 \%)$. En porcentaje de individuos colectados, los endoparásitos dominaron $(97 \% ; \mathrm{n}=259)$ frente a los ectoparásitos $(3 \% ; n=8)$. El 21.4\% del total de parásitos colectados $(\mathrm{N}=3)$ fueron formas maduras de metazoos de localización branquial y gastrointestinal y el $78.57 \%$ 
Cuadro 2. Tipo de especie, importancia específica, coeficiente de dispersión y tipo de distribución del componente comunitario parasítico de Sarda chiliensis de la zona costera de Chorrillos, Lima, Perú

\begin{tabular}{|c|c|c|c|c|}
\hline Parásito & $\begin{array}{l}\text { Tipo de } \\
\text { especie* }\end{array}$ & $\begin{array}{l}\text { Importancia } \\
\text { específica }\end{array}$ & $\begin{array}{l}\text { Coeficiente } \\
\text { de dispersión } \\
\text { (CD) }\end{array}$ & $\begin{array}{c}\text { Tipo de } \\
\text { distribución }\end{array}$ \\
\hline \multicolumn{5}{|l|}{ Copepoda } \\
\hline Caligus bonito & Satélite & 13 & $\mathrm{ND} * *$ & - \\
\hline \multicolumn{5}{|l|}{ Trematoda } \\
\hline Unitubulotestis pelamidys & Secundaria & 79 & 3.35 & agregada \\
\hline \multicolumn{5}{|l|}{ Cestoda } \\
\hline Sphyriocephalus tergestinus & Secundaria & 141 & 3.97 & agregada \\
\hline Tentacularia coryphaenae & Satélite & 9 & $\mathrm{ND} * *$ & - \\
\hline Nybelinia sp1 & Satélite & 3 & ND** & - \\
\hline Nybelinia sp2 & Satélite & 2 & $\mathrm{ND}^{* *}$ & - \\
\hline Adenocephalus pacificus & Satélite & 4 & ND** & - \\
\hline \multicolumn{5}{|l|}{ Nematoda } \\
\hline Anisakis sp 1 & Secundaria & 4 & ND** & - \\
\hline Anisakis sp2 & Secundaria & 78 & 1.22 & agregada \\
\hline Contracaecum sp & Satélite & 4 & ND** & - \\
\hline Hysterothylacium $\mathrm{sp}$ & Satélite & 22 & ND** & - \\
\hline \multicolumn{5}{|l|}{ Acantocephala } \\
\hline Corynosoma sp & Satélite & 7 & ND** & - \\
\hline Bolbosoma sp & Satélite & 2 & $\mathrm{ND}^{* *}$ & - \\
\hline Rhadinorhynchus sp & Satélite & 2 & ND** & - \\
\hline
\end{tabular}

$(\mathrm{N}=11)$ corresponde a formas larvarias. De los 100 peces evaluados, 42 (42\%) no presentaron infecciones parasitarias. Las infecciones monoespecíficas se encontraron en 29 hospederos (29\%), el biparasitismo en 15 $(15 \%)$, el triparasitismo en $10(10 \%)$ y el tetraparasitismo en 4 hospederos (4\%).

La P, IM y AM total de intención fueron del 58\%, 2.67 y 4.60, respectivamente. El Cuadro 1 muestra el sitio de infección, $P$, IM y AM de infección de las 14 taxa de metazoos parásitos encontrados en los 100 especímenes muestreados de $S$. chiliensis. La Figura 1 muestra el esquema de un espécimen de la costa central del Perú indicando sus macroparásitos agrupados en cinco hábitats: opérculo, branquia, estómago, intestino y superficie visceral.

Los tres parásitos con mayor importancia específica (prevalencia y abundancia media) fueron Sphyriocephalus tergestinus, Anisakis sp2 y Unitubulotestis pelamydis 
Cuadro 3. Frecuencia de dominancia de los parásitos componentes de Sarda chiliensis de la zona costera de Chorrillos, Lima, Perú

\begin{tabular}{lccc}
\hline Parásito & $\begin{array}{c}\text { Frecuencia de } \\
\text { dominancia }\end{array}$ & $\begin{array}{c}\text { Frecuencia de } \\
\text { dominancia de } \\
\text { dos especies }\end{array}$ & $\begin{array}{c}\text { Frecuencia de } \\
\text { dominancia } \\
\text { relativa }\end{array}$ \\
\hline Caligus bonito & 5 & 4 & 0.030 \\
Unitubulotestis pelamydis & 13 & 15 & 0.232 \\
Sphyriocephalus tergestinus & 29 & 20 & 0.408 \\
Tentacularia coryphaenae & 0 & 1 & 0.019 \\
Nybelinia $\mathrm{sp} 1$ & 0 & 1 & 0.011 \\
Nybelinia $\mathrm{sp} 2$ & 0 & 0 & 0.004 \\
Adenocephalus pacificus & 0 & 1 & 0.007 \\
Anisakis $\mathrm{sp}$ 1 & 0 & 1 & 0.007 \\
Anisakis $\mathrm{sp} 2$ & 17 & 14 & 0.176 \\
Contracaecum $\mathrm{sp}$ & 0 & 1 & 0.011 \\
Hysterothylacium $\mathrm{sp}$ & 2 & 1 & 0.075 \\
Corynosoma $\mathrm{sp}$ & 1 & 3 & 0.015 \\
Bolbosoma $\mathrm{sp}$ & 0 & 1 & 0.004 \\
Rhadinorhynchus $\mathrm{sp}$ & 0 & 0 & 0.004 \\
\hline
\end{tabular}

Cuadro 4. Valores de los coeficientes de correlación (r) usados para evaluar la relación entre la longitud total de Sarda chiliensis vs la prevalencia y abundancia de los metazoos parásitos más prevalentes de la zona costera de Chorrillos, Lima, Perú

\begin{tabular}{lcccc}
\hline Parásito & $\begin{array}{c}\mathrm{r}^{*} \\
\text { (Spearman) }\end{array}$ & $\mathrm{p}$ & $\begin{array}{c}\mathrm{r}^{* *} \\
\text { (Pearson) }\end{array}$ & $\mathrm{p}$ \\
\hline Unitubulotestis pelamydis & -1.00 & 0.001 & -0.013 & 0.90 \\
Sphyriocephalus tergestinus & -0.80 & 0.20 & -0.131 & 0.19 \\
Anisakis sp2 & 0.20 & 0.80 & 0.033 & 0.74 \\
Individuos & $\mathrm{NA}$ & $\mathrm{NA}$ & -0.104 & 0.30 \\
Riqueza & $\mathrm{NA}$ & $\mathrm{NA}$ & -0.062 & 0.54 \\
Abundancia total & $\mathrm{NA}$ & $\mathrm{NA}$ & -0.20 & 0.80 \\
\hline
\end{tabular}

$p=$ nivel de significancia, $r=$ coeficiente de correlación. $*=$ longitud total vs prevalencia. ${ }^{* *}=$ longitud total vs abundancia. NA = no aplica 
Cuadro 5. Valores de la prueba de $t$ de Student ( $\mathrm{t}$ ) y del estadístico de independencia Razón de Verosimilitud (RV) empleados para evaluar la relación entre el sexo de Sarda chiliensis y la abundancia y prevalencia de infección de los metazoos parásitos más prevalentes de la zona costera de Chorrillos, Lima, Perú

\begin{tabular}{lcccc}
\hline Parásito & $\mathrm{t}^{*}$ & $\mathrm{p}$ & $\mathrm{RV}^{* *}$ & $\mathrm{P}$ \\
\hline Unitubulotestis pelamydis & 1.11 & 0.27 & 1.57 & 0.20 \\
Sphyriocephalus tergestinus & 0.09 & 0.92 & 0.36 & 0.69 \\
Anisakis sp2 & 1.26 & 0.21 & 0.79 & 0.37 \\
Individuos & 0.63 & 0.52 & 0.60 & 0.43 \\
Riqueza & 0.64 & 0.51 & $\mathrm{NA}$ & $\mathrm{NA}$ \\
\hline
\end{tabular}

$\mathrm{p}=$ nivel de significancia. $\mathrm{NA}=$ no aplica. ${ }^{*}=$ comparar la abundancia media entre sexos. ${ }^{* *}=$ comparar prevalencia de infección entre sexos

Cuadro 6. Comparación de la longitud total de Sarda chiliensis parasitados y no parasitados para los metazoos parásitos más prevalentes de la zona costera de Chorrillos, Lima, Perú

\begin{tabular}{lcccc}
\hline Parásitos & $\begin{array}{c}\text { No parasitados } \\
(\mathrm{cm})\end{array}$ & $\begin{array}{c}\text { Parasitados } \\
(\mathrm{cm})\end{array}$ & $\mathrm{t}$ & $\mathrm{p}$ \\
\hline Unitubulotestis pelamydis & 40.01 & 39.59 & 0.53 & 0.59 \\
Sphyriocephalus tergestinus & 40.16 & 39.45 & 1.11 & 0.26 \\
Anisakis sp2 & 39.89 & 40.03 & -0.20 & 0.83 \\
Abundancia total & 40.22 & 39.73 & 0.80 & 0.42 \\
\hline
\end{tabular}

$\mathrm{t}=$ prueba de $\mathrm{t}$ de student. $\mathrm{p}=$ nivel de significancia

(Cuadro 2). Además, estos tres parásitos presentaron prevalencias entre 17 y $32 \%$, por lo que son consideradas especies secundarias, mientras que el resto de parásitos son considerados especies satélite, debido a su baja prevalencia $(<10 \%)$. El Cuadro 2 muestra en tres parásitos que el índice de dispersión mostró el típico patrón de distribución agregada con la siguiente secuencia de mayor a menor: S. tergestinus $>$ Anisakis sp2 $>U$. pelamydis. En 11 parásitos no se determinó el tipo de distribución por presentar prevalencias menores al 10\% (Caligus bonito, Tentacularia coryphaenae, Nybelinia sp1, Nybelinia sp2, Adenocephalus pacificus, Anisakis sp1, Contracaecum sp,
Hysterothylacium sp, Corynosoma sp, Bolbosoma sp y Rhadinorhynchus sp). La mayor frecuencia de dominancia y de dominancia relativa fueron para el cestodo $S$. tergestinus, para el nematodo Anisakis sp2 y el digeneo U. pelamydis (Cuadro 3).

La longitud total de $S$. chiliensis no presentó asociación entre la prevalencia y abundancia de dos parásitos (Cuadro 4), a excepción de $U$. pelamydis que observó relación entre la prevalencia y la talla del pez. No se observó relación entre la riqueza total de parásitos y la talla del pez, ni entre la abundancia total de parásitos y la longitud total del hospedero. 
Cuadro 7. Comparación entre los valores del factor de condición relativo $\left(\mathrm{K}_{\mathrm{n}}\right)$ entre Sarda chiliensis parasitados y no parasitados con los parásitos más prevalentes usando la prueba de student $(\mathrm{t})$ y la prueba de correlación $(\mathrm{r})$

\begin{tabular}{lccccccc}
\hline \multirow{2}{*}{ Parásito } & \multicolumn{7}{c}{$\mathrm{K}_{\mathrm{n}}$} \\
\cline { 2 - 7 } & Parasitados & $\begin{array}{c}\text { No } \\
\text { parasitados }\end{array}$ & $\mathrm{t}$ & $\mathrm{p}$ & $\mathrm{r}$ & $\mathrm{p}$ \\
\hline $\begin{array}{c}\text { Unitubulotestis } \\
\text { pelamydis }\end{array}$ & 0.86 & 1.02 & 2.06 & 0.01 & -0.25 & 0.010 \\
$\begin{array}{c}\text { Sphyriocephalus } \\
\text { tergestinus }\end{array}$ & 0.89 & 1.05 & 3.27 & 0.001 & -0.26 & 0.008 \\
$\begin{array}{c}\text { Anisakis sp2 } \\
\text { Abundancia }\end{array}$ & 0.87 & 1.05 & 3.53 & 0.001 & -0.32 & 0.001 \\
Riqueza & 0.88 & 1.16 & 7.04 & $<0.001$ & -0.38 & 0.000 \\
\hline
\end{tabular}

NA = no aplica

Cuadro 8. Índices de diversidad del componente comunitario y por sexo de Sarda chiliensis, índice que estima el número de especies de parásitos a encontrarse (Jack-1) de los metazoos parásitos de la zona costera de Chorrillos, Lima, Perú

\begin{tabular}{lccc}
\hline Índices & $\begin{array}{c}\text { Total de hospederos } \\
(\mathrm{n}=100)\end{array}$ & $\begin{array}{c}\text { Machos } \\
(\mathrm{n}=55)\end{array}$ & $\begin{array}{c}\text { Hembras } \\
(\mathrm{n}=45)\end{array}$ \\
\hline Riqueza & 14 & 11 & 9 \\
Individuos & 6.67 & 6.61 & 6.87 \\
Simpson & 0.45 & 0.43 & 0.47 \\
Shannon & 0.70 & 0.66 & 0.76 \\
Brillouin & 0.48 & 0.44 & 0.52 \\
Menhinick & 0.99 & 0.96 & 1.01 \\
Margalef & 0.82 & 0.79 & 0.86 \\
Equitabilidad & 0.81 & 0.80 & 0.83 \\
Berger-Parker & 0.64 & 0.64 & 0.64 \\
Chao 2 & 18 & - & - \\
\hline
\end{tabular}

El sexo de $S$. chiliensis no se encontró asociado con la prevalencia y abundancia de tres parásitos (Cuadro 5). Tampoco se encontró relación entre la riqueza total de parásitos y el sexo del pez (Cuadro 5).

No se observó relación entre la longitud total de S. chiliensis entre parasitados y no parasitados para los tres parásitos más prevalentes. Tampoco se observó relación entre la talla de parasitados y no parasitados con la abundancia total (Cuadro 6).

La comparación entre los valores de $\mathrm{k}_{\mathrm{n}}$ entre peces parasitados y no parasitados de $S$. chiliensis mostró diferencias para tres 


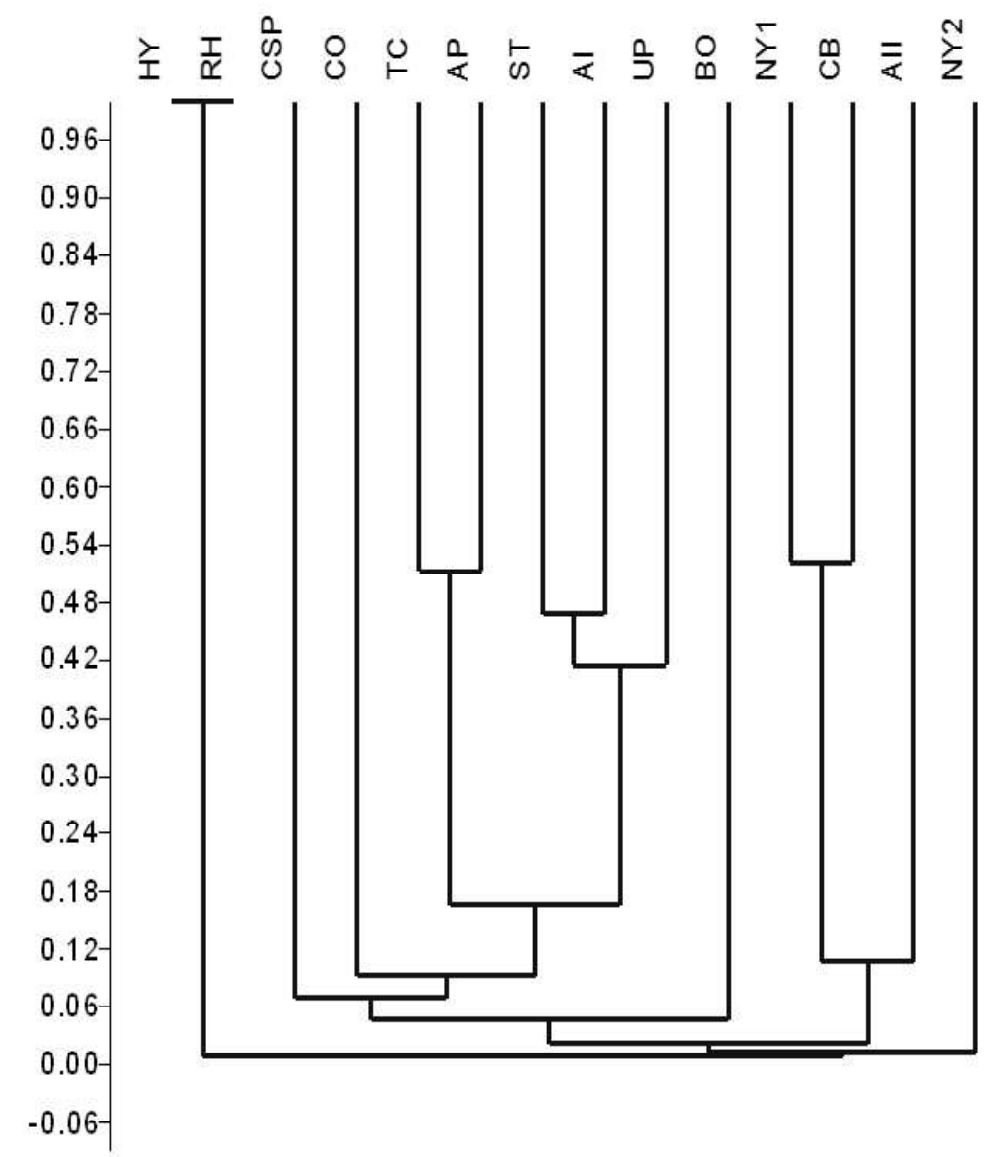

Figura 2. Dendrograma de similaridad con el índice cuantitativo de Morisita entre los parásitos compartidos entre los peces de catastrados de Sarda chiliensis de la zona costera de Chorrillos, Lima, Perú. ( $\mathrm{r}=0.92)$. $\mathrm{CB}=$ Caligus bonito; $\mathrm{UP}=$ Unitubulotestis pelamydis; $\mathrm{ST}=$ Sphyriocephalus tergestinus $; \mathrm{TC}=$ Tentacularia coryphaenae; $\mathrm{NY} 1=$ Nybelinia $\mathrm{sp} 1 ; \mathrm{NY} 2=$ Nybelinia $\mathrm{sp} 2 ; \mathrm{AP}=$ Adenocephalus pacificus; $\mathrm{AI}$ $=$ Anisakis sp 1 ;II $=$ Anisakis sp2; $\mathrm{HY}=$ Hysterothylacium $\mathrm{sp} ; \mathrm{CO}=$ Contracaecum $\mathrm{sp} ; \mathrm{CSP}=$ Corynosoma $\mathrm{sp} ; \mathrm{BO}=$ Bolbosoma $\mathrm{sp} ; \mathrm{RH}=$ Rhadinorhynchus sp

parásitos, presentándose mayores valores para los no parasitados (Cuadro 7). Además, se encontró diferencia para los valores de abundancia total entre el $\mathrm{k}_{\mathrm{n}}$ de parasitados y no parasitados. Se observó relación entre los valores de $\mathrm{K}_{\mathrm{n}}$ y la abundancia para los tres parásitos más prevalentes en el bonito del pacifico. También, se observó relación entre el $\mathrm{K}_{\mathrm{n}}$ vs la abundancia y riqueza total.
El Cuadro 8 muestra los valores de diversidad alfa según el total y el sexo en el componente comunitario parasitario. Hubo diferencias en la mayoría de los índices según el sexo del hospedero, siendo estos índices mayores en hembras que en machos. En el caso del componente comunitario se notó valores de dominancia según Berger-Parker y bajos según Simpson, e índices altos de 
equitabilidad y de Shannon, y bajos según Brillouin. Los valores de Chao-2 muestran que en el componente comunitario se requiere incrementar el tamaño de la muestra debido a que se espera encontrar hasta 18 especies $(22 \%+)$, y en el presente estudio solo se encontraron 14.

El dendrograma («análisis cluster») calculado con el índice cuantitativo de Morisita para comparar la asociación de los parásitos compartidos entre los peces catastrados muestra que se han formado cuatro grupos con similitud mayor al $40 \%$ en base a que los parásitos comparten los mismos individuos de peces hospederos muestreados (Figura 2). El primer grupo que mostró asociación parasitaria fue Hysterothylacium sp y Rhadinorhynchus sp. El segundo estuvo conformado por los cestodos T. coryphaenae y A. pacificus. El tercer grupo indicó una asociación parasitaria entre Nybelinia sp1 y C. bonito. Finalmente, el cuarto grupo de parásitos presentó una mayor asociación entre Anisakis sp1, S. tergestinus y $U$. pelamydis. El resto de macroparásitos mostró escasa asociación parasitaria en el dendrograma (Figura 2).

\section{Discusión}

En la presente investigación se muestra el predominio en la abundancia numérica y riqueza de taxas de los endoparásitos sobre los ectoparásitos (copépodos). Alves y Luque (2006) reportaron la dominancia de endoparásitos en cuatro especies de escómbridos: Euthynnus alleteratus (Rafinesque, 1810), Katsuwonus pelamis (Linnaeus, 1758), Scomber scombrus (Linnaeus, 1758) y Scomberomurus brasiliensis Collette, Russo and ZavalaCamim, 1978, así como la dominancia de ectoparásitos representados por los copépodos en Sarda sarda (Bloch, 1793) del estado de Rio de Janeiro (Brasil). Sin embargo, en el presente estudio, los copépodos representan solo el 3\% del total de parásitos colectados en el componente comunitario de S. chiliensis. Además, Alves y Luque (2006) señalan hasta tres especies de copépodos en S. sarda: C. bonito Wilson C.B, 1905; Caligus pelamydis Krøyer, 1863 y Lernanthropus sp, con prevalencias del 30 , 83.4 y $3.3 \%$, respectivamente. En $S$. chiliensis en el presente estudio solo se registró una especie $(C$. bonito) con una baja prevalencia $(\mathrm{P}=2 \%)$. Estas diferencias en prevalencias y riqueza pueden ser atribuidas a las condiciones ambientales del área geográfica de donde provienen los hospederos (Ibagy y Sinque, 1985).

En el ámbito sudamericano, la dominancia de endoparásitos para otras comunidades de peces escómbridos ha sido bien documentada. Así, Alves et al. (2003) reportaron la dominancia en la riqueza y abundancia de endoparásitos en $S$. japonicus de la zona costera del estado de Rio de Janeiro (Brasil). Oliva et al. (2008) resaltan el predominio de endoparásitos sobre ectoparásitos en $S$. japonicus para la zona de Antofagasta (Chile) e Islas Madeira (Portugal). Cruces et al. (2014) señalan para $S$. japonicus la dominancia de endoparásitos en el puerto de Chicama, La Libertad, Perú. Justo y Kohn (2014) indican para Thunnus atlanticus (Lesson, 1831) un mayor número de endoparásitos. Según Tam et al. (2008), la dominancia de endoparásitos en el componente comunitario parasítico de peces marinos puede ser asignada al comportamiento trófico de los peces hospederos por ser organismos de hábitos principalmente ictiófagosomnívoros, que incluyen una amplia gama de invertebrados acuáticos que pueden actuar como hospederos intermediarios en el ciclo de vida de varios endohelmintos. Alves y Luque (2006) atribuyen la dominancia de endoparásitos al hábito alimenticio, al nivel trófico y a la distribución geográfica de los peces hospederos. La mayoría de estudios sobre comunidades de parásitos en peces marinos de la costa del Perú muestran un patrón de dominancia de endoparásitos (Iannacone y Alvariño, 2008; Iannacone et al., 2010a; Iannacone et al., 2012; Nacari y 
Sánchez, 2014; Chero et al., 2014a,b,c,d; Iannacone et al., 2015).

Los trematodos de la familia Didymozoidae son parásitos comunes en teleósteos marinos, principalmente escómbridos y rara vez son encontrados en peces de agua dulce (Pascual et al., 2006; Justo y Kohn, 2011; Radujkoviæ y Šundiæ, 2014; Chero et al., 2015). Estos trematodos representan un grupo diferente de macroparásitos por presentar características morfológicas altamente adaptadas a diferentes hábitats (Justo y Kohn, 2011). Køie y Lester (1985) señalan que el ciclo biológico de los didimozoideos presenta cuatro hospederos, con los moluscos, los crustáceos y los peces pequeños como los primeros, segundos y terceros hospederos intermediarios, respectivamente y los peces depredadores, como los escómbridos, como hospederos definitivos. La mayor riqueza y abundancia de este grupo de parásitos se encuentra en el océano pacifico, siendo la región de Hawái y la región neotropical las que presentan la mayor diversidad (Nikolaeva, 1981).

Justo y Kohn (2011) señalan que en el ensamblaje comunitario de metazoos parásitos de peces escómbridos los digeneos de la familia Didymozoidea son los más abundantes y prevalentes. Además, señalan que este patrón es muy frecuente en peces escómbridos de Brasil. Cruces et al. (2014) observaron este tipo de patrón al analizar los metazoos parásitos de $S$. japonicus en el puerto de Chicama (Perú) registrando un total de cinco taxa de didymozoideos. Sin embargo, en $S$. chiliensis solo se registró una especie de didymozoideo (U. pelamydis) con una $\mathrm{P}$ del $62 \%$. En una especie congenérica de la costa de Brasil, se registró en $S$. sarda a una especie de didymozoideo ( $U$. pelamydis) con una $\mathrm{P}$ del 20\% (Alves y Luque, 2006). Probablemente, las diferencias en la prevalencia de $U$. pelamydis en estos dos hospederos ( $S$. sarda y $S$. chiliensis) sea el resultado de presentar preferencias en sus ítems alimentarios, dado que $S$. chiliensis presenta como principal ítem presa a $E$. ringens, anchoveta, mientras que el bonito del atlántico $S$. sarda presenta como principal ítem alimentario a las anchoas y secundariamente a las anchovetas (Collette, 2003). Asimismo, el ciclo biológico de U. pelamydis no es conocido, pero es probable que $E$. ringens actúe como tercer hospedero intermediario.

El copépodo C. bonito; los cestodos $T$. coryphaenae, Nybelinia sp1, Nybelinia sp2 y A. pacificus, los nematodos Anisakis sp1, Contracaecum sp e Hysterothylacium sp y los acantocefalos Corynosoma sp, Bolbosoma sp y Rhadinorhynchus sp presentaron prevalencia menores al $10 \%$ por lo que en este trabajo son consideradas especies satélites. Iannacone et al. (2010a,b) asignan las bajas prevalencias en las comunidades de macroparásitos de peces marinos a las condiciones ambientales del área de colecta, principalmente a los factores ambientales abióticos de la Provincia Faunística Peruana, al evento de El Niño y al fenómeno de surgencia, señalando también que pueden ser atribuidas al estrecho rango de tallas analizadas $(32-48 \mathrm{~cm})$, ya que se puede alcanzar tallas de $79 \mathrm{~cm}$ de LT (Oliva y Luque,1998).

Una característica encontrada durante el periodo de muestreo señala que las formas larvarias de endohelmintos (T. coryphaenae, Nybelinia sp1, Nybelinia sp2, S. tergestinus, A. pacificus, Anisakis sp1, Anisakis sp2, Contracaecum sp, Hysterothylacium sp, Corynosoma sp y Bolbosoma sp) son los principales componentes en la comunidad parasítica de $S$. chiliensis. La presencia de larvas de macroparásitos en el presente estudio puede ser considerada un reflejo del nivel trófico de $S$. chiliensis que actuaría en una escala intermedia en la cadena alimentaria marina, como consecuencia de un hábitat epipelágico. Además, Alves et al. (2003) señalan que este patrón estaría relacionado al comportamiento alimenticio de los peces escómbridos como $S$. chiliensis, pues esta especie de hospedero se alimenta de peces planctívoros, principalmente anchoveta. En la costa peruana los cestodos $T$. 
coryphaenae y Nybelinia sp, presentan como hospederos definitivos a peces elasmobranquios, mientras que $A$. pacificus presenta a mamíferos marinos como hospederos definitivos (Tantaleán y Huiza, 1994; Sarmiento et al., 1999; Chero et al., 2014a). No se conoce el hospedero definitivo de $S$. tergestinus en el Perú; sin embargo, se conoce para otras localidades geográficas (Beveridge y Campbell, 1996; Jakob y Palm, 2006; Chero et al., 2015).

En el presente estudio se encontraron larvas del tercer estadio de Anisakis sp1, Anisakis sp2, Hysterothylacium sp y Contracaecum sp. Estas se encontraron en una muy baja prevalencia y abundancia parasitaria, con excepción de Anisakis sp2 que se encuentra entre las especies más prevalentes. Estas especies de helmintos tienen implicaciones zoonóticas junto a otras especies de anisákidos, al ser responsables de infecciones humanas causadas por la ingesta de peces crudos o inadecuadamente cocidos (Iannacone et al., 2010b; MendozaCruz et al., 2013). Osanz-Mur (2001) señala que los niveles de prevalencia como los grados de parasitación de anisákidos son muy variables, y dependen de factores como la especie de pez, zona geográfica, época del año y características individuales de cada pez hospedero. Esto podría explicar la variación observada en las prevalencia de estos nematodos a través del tiempo, pues en $S$. chiliensis se observaron valores bajos de infección por Anisakis sp2 en la época de invierno.

Los nematodos Anisakis sp1, Anisakis sp2 y Contracaecum sp presentan como hospederos definitivos a mamíferos marinos y aves piscívoras y los seres humanos pueden llegar a actuar como hospederos accidentales al ingerir peces infestados (Tantaleán y Huiza, 1994; Sarmiento et al., 1999; Chero et al., 2014a), mientras que los estadios larvales han sido recuperados de una gran variedad de peces y moluscos marinos de importancia económica (Tantaleán y Huiza, 1994; Sarmiento et al., 1999; Chero et al., 2014a,b,c; Cruces et al., 2014). Las especies de Hysterothylacium sp como larvas han sido recuperadas de $S$. chiliensis y como adultos de Coryphaena hippurus Linnaeus, 1758 (Sarmiento et al., 1999; Gómez-Puerta, 2014).

Los acantocefalos de la familia Polymorphidae son endoparásitos con ciclos de vida complejo, presentan a invertebrados acuáticos (anfipodos, decápodos y eufásidos) como hospederos intermediarios y a una amplia variedad de peces teleósteos como hospederos paraténicos, mientras que las aves piscívoras y mamíferos marinos actúan como hospederos definitivos (García-Varela et al., 2005). En el presente estudio se ha registrado a los juveniles de Corynosoma sp y Bolbosoma sp con prevalencias del 3 y $1 \%$, respectivamente. Iannacone et al. (2015) señalan que la presencia de cistacantos de Corynosoma en el ecosistema marino de Chorrillos, Lima, parece estar relacionada con las redes tróficas entre los microcrustáceos, la especie parásita indicada, los peces paraténicos involucrados como $S$. chiliensis y los pinnípedos, como hospederos definitivos. Esta es la primera vez que se hace mención a Corynosoma sp en $S$. chiliensis, especie que actuaría como hospedero paraténico.

El género Bolbosoma incluye especies con potencial zoonótico y que pueden infectar ocasionalmente a humanos (Tada et al., 1983). Bolbosoma sp es un nuevo registro para el Perú y $S$. chiliensis es un nuevo hospedero. El rol de Bolbosoma sp en $S$. chiliensis no está claro pero debido a su baja prevalencia $(\mathrm{P}=1 \%)$, esta especie podría haber infectado de forma accidental a $S$. chiliensis.

Los peces parasitados por U. pelamydis, S. tergestinus y Anisakis sp2 presentaron valores más bajos de $\mathrm{k}_{\mathrm{n}}$ en comparación con los no parasitados. Estos datos concuerdan con lo propuesto por Graça et al. (2013), quienes mencionan que los peces más infectados presenten valores más bajos de $\mathrm{K}_{\mathrm{n}}$. Este es un indicador cuantitativo para detectar 
parásitos que afectan el bienestar de los peces hospederos. Variaciones en el $\mathrm{K}_{\mathrm{n}}$ indican cambios en el ambiente, carencia de alimento o parasitismo (Silva et al., 2011). La dependencia entre el crecimiento y ganancia de peso $\left(\mathrm{k}_{\mathrm{n}}\right)$ y el parasitismo en $S$. chiliensis podría deberse a la alta patogenicidad de los parásitos, que influyen en la correlación $\mathrm{k}_{\mathrm{n}}$ ' parasitismo (Moreira et al., 2010). Algunos investigadores han encontrado una asociación entre el $K_{n}$ y el parasitismo por macroparásitos en peces del litoral peruano, evidenciando un efecto en la salud del pez hospedero (Iannacone y Alvariño, 2013; Cruces et al., 2014).

\section{Literatura Citada}

1. Alves DR, Luque, JL, Abdallah, V.D. 2003. Metazoan parasites of chub mackerel, Scomber japonicus Houttuyn (osteichthyes: scombridae), from the coastal zone of the State of Rio de Janeiro, Brazil. Rev Bras Parasitol Vet 12: $164-170$.

2. Alves DR, Luque, JL. 2006. Ecologia das comunidades de metazoários parasitos de cinco espécies de escombrídeos (Perciformes: Scombridae) do litoral do Estado do Rio de Janeiro, Brasil. Rev Bras Parasitol Vet 15: 167-181.

3. Amin OA. 2013. Classification of the Acanthocephala. Folia Parasitol (Praha) 60: 273-305. doi: 10.14411/fp.2013.031

4. Bego NM, Von Zuben CJ. 2010. Métodos quantitativos em parasitologia. Jaboticabal, Brasil: FUNEP. 72 p.

5. Baer J. 1969. Diphyllobothrium pacificum, a tapeworm from sea lions endemic in man along the coastal area of Peru. J Fish Res Bd Canada 26: 717723. doi: 10.1139/f69-071

6. Beveridge I, Campbell R.A. 1996. New records and descriptions of trypanorhynch cestodes from Australian fishes. Rec South Aust Mus 29: 1-22.
7. Bursey CR, Goldberg SR, Pamarlee JR. 2001. Gastrointestinal helminths of 51 species of anurans from Reserva Cuzco Amazónico, Peru. Comp Parasitol 68:21-35.

8. Bush AO, Lafferty KD, Lotz JL, Shostak AW. 1997. Parasitology meets ecology on its own terms: Margolis et al. revisited. J Parasitol 83: 575-583. doi: $10.2307 / 3284227$

9. Chero J, Cruces C, Iannacone J, Sáez $G$, Alvariño L, Rodríguez C, Rodríguez H, et al. 2014a. Índices parasitológicos de la merluza peruana Merluccius gayi peruanus (Ginsburg, 1954) (Perciformes: Merlucciidae) adquiridos del terminal pesquero de Ventanilla, Callao, Perú. Neotrop Helminthol 8: 141-162.

10. Chero J, Iannacone J, Cruces C, Sáez G, Alvariño L. 2014b. Comunidad de metazoos parásitos de la corvina Cilus gilberti (Abbott, 1899) (Perciformes: Sciaenidae) en la zona costera de Chorrillos, Lima, Perú. Neotrop Helminthol 8: 163-182.

11. Chero J, Sáez G, Iannacone J, Aquino W. 2014c. Aspectos ecológicos de los helmintos parásitos de lorna Sciaena deliciosa (Tschudi, 1846) (Perciformes: Sciaenidae) adquiridos del terminal pesquero de Ventanilla, Callao, Perú. Neotrop Helminthol 8: 59-76.

12. Chero J, Cruces C, Iannacone J, Saez G, Alvariño L. 2014d. Helminth parasites of Anisotremus scapularis (Tschudi, 1846) (Perciformes: Haemulidae) «Peruvian grunt» acquired at the Fishing Terminal of Villa Maria del Triunfo, Lima, Peru. Neotrop Helminthol 8: 411-428.

13. Chero J, Cruces C, Iannacone J, Sáez G, Sánchez L, Minaya D, Alvariño L, et al. 2015. First record of Unitubulotestis pelamydis (Trematoda: Didymozoidae) and Sphyriocephalus tergestinus (Cestoda: Sphyriocephalidae) in eastern pacific bonito, Sarda chiliensis (Perciformes: Scombridae) in Peru. Neotrop Helminthol 9:313-323. 
14. Chirichigno N, Cornejo RM. 2001. Catálogo comentado de los peces marinos del Perú. Callao, Perú: Instituto del Mar del Perú. 314 p.

15. Cruces C, Chero J, Iannacone J, Diestro A, Sáez G, Alvariño L. 2014. Metazoans parasites of «chub mackerel» Scomber japonicus Houttuyn, 1782 (Perciformes: Scombridae) at the port of Chicama, La Libertad, Peru. Neotrop Helminthol 8: 357-381.

16. Collette B, Nauen C. 1983. An annotated and illustrated catalogue of tunas, mackerels, bonitos and related species known to date. En : FAO Species Catalogue. Scombrids of the world. Vol 2. Rome: FAO. [Internet]. Available in: http://www.fao.org/docrep/ 009/ac478e/ac478e00.htm

17. Collette BB. 2003. Family Belonidae Bonaparte 1832 - needlefishes. Calif Acad Sci Annotated Checklists of Fishes 16: 1-22.

18. Collette B, Acero A, Canales-Ramírez $C$, Cárdenas G, Carpenter KE, Di Natale A, Guzman-Mora A, et al. 2011. Sarda chiliensis. The IUCN Red List of Threatened Species 2011. [Internet]. Available in: http://dx.doi.org/ 10.2305/IUCN.UK.2011-2.RLTS. T170352A6763952.en

19. Escalante H. 1983. Larvas plerocercoides de Diphyllobothriidae Lue, 1910: Hallazgo en peces marinos de consumo humano en la costa peruana. Bol Chile Parasit 38: 50-52.

20. García-Varela M, Aznar FJ, PérezPonce de León G, Piñero $D$, Laciette JP. 2005. Molecular phylogeny of Corynosoma Luhe, 1904 (Acanthocephala), based on $5.8 \mathrm{~S}$ and internal transcribed spacer sequences. J Parasitol 91:345-352.

21. Gibson DJ, Jones A, Bray RA. 2002. Keys to the Trematoda. Vol 1. London: CAB International and the Natural History Museum. $521 \mathrm{p}$.

22. Gómez-Puerta LA. 2014. Primeros registros de Hysterothylacium pelagicum (Anisakidae) y Toxocara alienata
(Toxocaridae) en el Perú. Rev Peru Biol 21: 171-174. doi: 10.15381/rpb.v21i2. 9820

23. Graça RJ, Costa APL, Takemoto RM. 2013. Ecological aspects of monogenean gill parasites (Platyhelminthes) from Hoplias aff. malabaricus (Bloch, 1794) (Pisces, Erythrinidae) in a neotropical floodplain. Neotrop Helminthol 7: 105-116.

24. Heinz ML, Dailey MD. 1974. The Trypanorhyncha (Cestoda) of elasmobranch fishes from southern California and Northern Mexico. Proc Helminthol Soc Wash 41: 161-169.

25. lannacone J, Alvariño L. 2008. Influencia del tamaño y sexo de Peprilus medius (Peters) (Stromateidae: Perciformes) capturados en Chorrillos, Lima, Perú, sobre su comunidad parasitaria. Neotrop Helminthol 2: 62-70.

26. Iannacone J, Alvariño L. 2009. Catastro de la fauna endoparasitaria de la pota Dosidicus gigas (Cephalopoda) en el norte del Perú. Neotrop Helminthol 3: 89-100.

27. Iannacone J, Alvariño L, Alayo M. 2008. Aspectos ecológicos de los metazoos parásitos de Callichirus seilacheri (Bott 1955) (Decapoda, Callianassidae) en Lima, Perú. Neotrop Helminthol 2: 9-17.

28. Iannacone J, Cárdenas-Callirgos J, Alvariño L. 2010a. Ecología comunitaria de los parásitos de la chilindrina Stromateus stellatus (Cuvier, 1829) (Perciformes: Stromateidae) de la zona costera de Chorrillos, Lima, Perú. Neotrop Helminthol 4: 159-167.

29. Iannacone J, Morón L, Guizado S. 2010b. Variación entre años de la fauna de parásitos metazoos de Sciaena deliciosa (Tschudi, 1846) (Perciformes: Sciaenidae) en Lima, Perú. Lat Am J Aquat Res 38: 218-226. doi: 10.3856/ vol38-issue2-fulltext-6

30. Iannacone J, Alvariño L. 2011. Aspectos cuantitativos de los parásitos del pejesapo Sicyases sanguineus (Müller \& Troshel, 1843) (Perciformes: 
Gobiesocidae) de la zona costera de Chorrillos, Lima, Perú. Neotrop Helminthol 5: 56-72.

31. Iannacone J, Sánchez V, Olazábal N, Salvador C, Alvariño L, Molano J. 2012. Ecological indices of parasites of Scartichthys gigas (Steindachner, 1876) (Perciformes: Blenniidae) of the coasts of Lima, Peru. Neotrop Helminthol 6: 191-203.

32. Iannacone J, Alvariño L. 2012. Microecology of the monogenean Mexicana sp. on the gills of Anisotremus scapularis (Tschudi, 1846) (Osteichthyes, Haemulidae) of the marine coast of Lima, Peru. Neotrop Helminthol 6: 277-285.

33. Iannacone J, Alvariño L. 2013. Parasitological indices of Pacific pomfret Brama japonica Hilgendorf, 1878 (Osteichthyes, Bramidae) acquired at fishing terminal of Chorrillos Lima, Peru. Neotrop Helminthol 7: 117-132.

34. Iannacone J, Alvariño L, Chero J, Sáez G 2015. Comunidad parasitaria de cabinza Isacia conceptionis (Cuvier \& Valenciennes, 1830) (Perciformes: Haemulidae) en la zona de Chorrillos, Lima, Perú. Rev Inv Vet Perú 26: 96110. doi: 10.15381/rivep.v26i1.10943

35. Ibagy $A S$, Sinque C. 1985. Distribuição de ovos e larvas de Sciaenidae (Teleostei, Perciformes) na região costeira do Rio Grande do Sul, Brasil. Sciaenidae. Arq Biol Tecnol 38: 249-270.

36. Ilave V. 1964. Tremátodes intestinales de Sarda chiliensis «bonito». Tesis Bach. Lima: Univ Nacional Mayor de San Marcos. 19 p.

37. Jakob E, Palm HW. 2006. Parasites of commercially important fish species from the southern Java coast, Indonesia, including the distribution pattern of trypanorhynch cestodes. Verh Ges Ichthyol Bd 5: 165-191.

38. Jones A, Bray RA, Gibson DJ. 2005. Keys to the Trematoda. Vol 2. London: CAB International. $768 \mathrm{p}$.

39. Justo MCN, Kohn A. 2011. New data on three species of Koellikerioides
(Digenea, Didymozoidae) in Brazilian tuna fish. Neotrop Helminthol 5: 113-123.

40. Justo MCN, Kohn A. 2014. Monogenoidea and Digenea parasites of Thunnus atlanticus (Perciformes, Scombridae) from Rio de Janeiro coast, Brazil. Neotrop Helminthol 8: 339-348.

41. Koie M, Lester, RJG. 1985. Larval Didymozoids (Trematoda) in fishes from Moreton Bay, Australia. Proc Helminthol Soc Wash 52: 196-203.

42. Lazarte R, Córdova E. 1987. Estudio de un parásito de Sarda chiliensis chiliensis (Cuvier) proveniente de la costa sur del Perú. Res. X Congreso Latinoamericano de Microbiología. Trujillo, Perú. p 82.

43. Longart RY, Acosta V, Parra B, Lista M. 2011. Aspectos biométricos de Hemirhamphus brasiliensis (Pisces: Hemirhamphidae), Isla de Cubagua, Venezuela. Zoot Trop 29: 385-398.

44. Luque JL, Iannacone J. 1991. Some monogenoidea parasitic on Peruvian marine fishes, with description of Anoplocotyloides chorrillensis new species and new records. Mem Inst Oswaldo Cruz 86: 425-428. doi: 10.1590/ S0074-02761991000400008.

45. Mendoza-Cruz M, Valles-Vega I, LozanoCobos H, Gómez del PradoRosas MC. 2013. Parasite fauna of Paranthias colonus (Valenciennes, 1846) from El Sargento, Baja California, México. Neotrop Helminthol 7: 13-28.

46. Moravec F. 2006. Dracunculoid and Anguillicoloid nematodes parasitic in vertebrates. Prague: Academia. $634 \mathrm{p}$.

47. Moreira LHA, Yamada FH, Ceschini TL, Takemoto RM, Pavanelli C. 2010. The influence of parasitism on the relative condition factor $(\mathrm{Kn})$ of Metynnis lippincottianus (Characidae) from two aquatic environments: the upper Parana river floodplain and Corvo and Guairacá rivers, Brazil. Acta Sci Biol Sci 32: 8386. doi: 10.4025/actascibiolsci.v32i1.3668

48. Moreno E. 2001. Métodos para medir la biodiversidad. M\&T - Manuales y Tesis SEA. CYTED. México. 84 p. 
49. Nikolaeva VM. 1981. [Trematodes Didymozoidea: fauna, distribution, biology.] In: Symposium on Parasitology and Pathology of Marine Organisms. Leningrad [in Russian].

50. Ñacari L, Sánchez L. 2014. Helminth fauna of Peprilus snyderi Gilbert \& Starks, 1904 (Stromateidae) of Chorrillos fishmarket, Lima, Peru. Neotrop Helminthol 8: 1-17.

51. Oliva ME, Luque JL. 1998. Metazoan parasite infracommunities in five sciaenid from the central Peruvian coast. Mem Inst Oswaldo Cruz 93: 175-180. doi: 10.1590/S0074-02761998000200007

52. Oliva ME, Valdivia IM, Costa G, Freitas $N$, Pinheiro de Carvalho MA, Sánchez L, Luque JL. 2008. What can metazoan parasites reveal about the taxonomy of Scomber japonicus Houttuyn in the coast of South America and Madeira Islands? J Fish Biol 72: 545554. doi: 10.1111/j.1095-8649.2007. 01725.x

53. Osanz-Mur AC. 2001. Presencia de larvas de Anisákidos (Nematoda: Ascaridoidea) en pescado de consumo capturado en la zona pesquera de Tarrogona. Tesis de Doctorado. Barcelona, España: Univ Autónoma de Barcelona. $142 \mathrm{p}$.

54. Pascual S, Abollo E, Azevedo C. 2006. Host parasite interaction of a muscleinfecting didymozoid in the Atlantic mackerel Scomber scombrus L. ICES IJMS 63: 169-175. doi: 10.1016/ j.icesjms.2005.08.010

55. Petrochenko VI. 1971. Acanthocephala of domestic and wild animals. Vol. II. Jerusalem: Israel Program for Scientific Translations. $478 \mathrm{p}$.

56. Radujkoviae BM, Šundice D. 2014. Parasitic flatworms (Platyhelminthes: Monogenea, Digenea, Cestoda) of fishes from the Adriatic Sea. Natura Montenegrina 13: 7-280. doi: 10.13140/ RG.2.1.1401.5448

57. Rego AA, Vicente JJ, Ibañez HN. 1968. Sobre dois novos parasitos de peixe da costa de Peru (Cestoda:
Tetraphyllidea). Mem Inst Oswaldo Cruz 66: 145-149. doi: 10.1590/S007402761968000200002

58. Rodhe K, Hayward C, Heap M. 1995. Aspects of the ecology of metazoan ectoparasites of marine fishes. Int $\mathbf{J}$ Parasitol 25: 945-970.

59. Ruelas N, Córdova E. 1988. Algunos trematodos parásitos de peces de la zona sur del mar peruano. Res IX Congreso Nacional de Biologia. Piura, Perú. p 176.

60. Sarmiento L, Tantaleán M, Huiza A. 1999. Nemátodos parásitos del hombre y de los animales en el Perú. Rev Peru Parasitol 14: 9-65.

61. Silva OAM, Tavares-Dias $M$, Fernandes JS. 2011. Helminthes parasitizing Semaprochilodus insignis Jardine, 1841 (Osteichthyes: Prochilodontidae) from the central Amazonia (Brazil), and their relationship with the host. Neotrop Helminthol 5: 225-233.

62. Schmidt GD. 1986. Handbook of tapeworm identificatiov. Boca Raton, Florida: CRC Press. 675 p.

63. Tada I, Otsuji Y, Kamiya H, Mimori T, Sakaguchi Y, Makizumi S. 1983. The first case of a human infected with an Acanthocehalan parasite, Bolbosoma sp. J Parasitol 69: 205-208. doi: 10.2307/ 3281300

64. Tam J, Marc H, Taylor MH, Blaskovic V, Espinoza P, Michael-Ballón R, Díaz E, et al. 2008. Trophic modeling of the Northern Humboldt Current Ecosystem. Part I: Comparing trophic linkages under La Niña and El Niño conditions. Prog Oceanogr 79: 352-365. doi: 10.1016/ j.pocean.2008.10.007

65. Tantaleán M, Huiza A. 1994. Sinopsis de los parásitos de peces marinos de la costa peruana. Biotempo 1: 53-101.

66. Tantaleán M, Sánchez L, Gómez L, Huiza A. 2005. Acantocéfalos del Perú. Rev Peru Biol 12: 83-92.

67. Yamaguti S. 1971. Synopsis of digenetic trematodes of vertebrates. Vol I-II. Tokio: Keigaku Publ Co. 1074 p. 